\section{Deprogramming stroke}

\section{By Lev Osherovich, Senior Writer}

Canadian researchers have taken an entirely new tack on preventing neurotoxicity in acute ischemic stroke by targeting a pathway previously linked to cholesterol metabolism. ${ }^{1}$ The University of British Columbia team suggests that a set of proteins that monitors cholesterol levels in the liver plays a completely different role in the brain-relaying a proapoptotic signal triggered by excessive glutamate, a key feature of ischemic stroke.

In ischemic stroke, blockage of cerebral arteries by blood clots leads to oxygen deprivation and triggers neuronal hyperstimulation by glutamate, an excitatory neurotransmitter that is released by oxygen-starved cells. Although lack of oxygen can cause rapid cell death near the site of ischemia, glutamate can also spread to nearby areas and cause apoptosis in otherwise welloxygenated cells.

\section{"Lots of extracellular glutamate leads to calcium influx and cell death. Acute cell death is difficult to prevent, but with programmed cell death there is some hope of intervention."}

-Yu Tian Wang, The University of British Columbia
Thus, rapidly shutting down the glutamate toxicity cascade could stop small ischemic events from snowballing into massive strokes.

"Lots of extracellular glutamate leads to calcium influx and cell death," said Yu Tian Wang, professor of neurology at The University of British Columbia (UBC) and lead author of the study. "Acute cell death is difficult to prevent, but with programmed cell death there is some hope of intervention."

To block neurotoxicity in stroke, researchers have focused on NMDAR, which binds to glutamate and sets off intracellular events ficult because it acts early in the neurotoxic cascade and may no longer e relevant by the time stroke has set in.

Wang's team thus sought to identify and cut off components of the pathway downstream of NMDAR. The group profiled the activity of a panel of 345 transcription factors in cultured rat neurons treated with NMDA, which mimics glutamate overstimulation, and identified 21 proteins that became strongly activated. Among these was sterol regulatory element binding transcription factor 1 (SREBF1; SREBP1), a transcription factor better known as the master regulator of cholesterol biosynthesis. ${ }^{2}$

Building on previous evidence that ischemic brains have altered lipid levels compared with healthy control brains, ${ }^{3}$ Wang's team worked out the mechanism of SREBP1 activation in stroke. The team found that in cultured rat neurons, NMDA caused SREBP1 to travel from the cytoplasm to the nucleus. There, the tran-
Figure 1. New pathway to neuron death in stroke. Taghibiglou et al. propose a new mechanism for how neurons die after ischemia. The pathway involves activation of sterol regulatory element binding transcription factor 1 (SREBF1; SREBP1).

Under ischemic conditions, excessive extracellular glutamate activates neuronal NMDARs [a] and induces proteasomemediated degradation of insulin induced gene 1 (INSIG1) at the surface of the endoplasmic reticulum [b]. After INSIG1 is degraded [c], SREBP1 and its carrier protein, SREBF chaperone (SCAP), travel by vesicles [d] to the Golgi apparatus, in which SREBP1 becomes activated [e]. Active SREBP1 enters the nucleus [ $\mathbf{f}$ ] and turns on the transcription of genes that cause apoptosis [g].

Blocking the pathway therefore could be useful for preventing neuron death after ischemia. Taghibiglou et al. blocked SREBP1 activation with a peptide inhibitor of INSIG1 degradation. Kamisuki et al. have shown that a small molecule, fatostatin, that binds to SCAP can also prevent SREBP1 activation. 


\section{Box 1. Fat chance.}

Findings by Canadian researchers that the sterol regulatory element binding transcription factor 1 (SREBF1; SREBP1) pathway has a role in acute ischemic stroke ${ }^{1}$ could provide a new indication for an SREBP1-modulating small molecule developed by a team at Baylor College of Medicine. ${ }^{6}$

Fatostatin was discovered by Motonari Uesugi, associate professor of biochemistry and molecular biology, and Salih Wakil, professor in the same department. Wakil said the discovery stemmed from the team's efforts to turn down lipid synthesis, which is SREBP1's primary role in the liver.

In 2003, Uesugi screened a small molecule library for molecules that interfered with fat production in cell culture and identified fatostatin as the strongest lead compound. ${ }^{7}$ This August, the researchers published in Chemistry \& Biology that fatostatin blocked lipid synthesis and weight gain in a mouse model of obesity.

The study showed that fatostatin binds to SREBF chaperone (SCAP), a chaperone that helps SREBP1 travel from the endoplasmic reticulum (ER) to the Golgi apparatus when cholesterol levels are low (see Figure 1, "New pathway to neuron death in stroke"). Once in the Golgi, SREBP1 dissociates from SCAP, becomes activated by a protease and travels to the nucleus to transcribe genes needed to make lipids.

Although fatostatin's precise mechanism is unclear, its interaction with SCAP blocks the degradation of an ER protein called insulin induced gene 1 (INSIG1) that confines both SCAP and SREBP1 to the ER.

"INSIG1 and SCAP both respond to cholesterol and cooperate to keep SREBP1 in the ER" when cholesterol levels are high, said Wakil. Fatostatin thus simulates conditions of high lipid levels and prevents cells from making more cholesterol.

Although the main utility of fatostatin could be in treating metabolic diseases, Wakil believes that the Nature Medicine report by researchers at The University of British Columbia presents an opportunity for using fatostatin in stroke.

"Most of our work was in the liver, but fatostatin absolutely could be useful for ischemia," said Wakil. "We're interested in testing its effects in ischemic cells."

He said his lab is running toxicology and pharmacodynamic studies of fatostatin in rats and hopes to put the molecule in the clinic for dyslipidemia.

Uesugi and Wakil have filed for patents on fatostatin and its analogs, which are available for licensing. $\quad-L Z O$ scription factor turned on the expression of genes involved in both lipid metabolism and apoptosis.

Wang's team found that SREBP1 is required for the neurotoxic effects of NMDAR activation. Cultured neurons treated with cholesterol, which inhibits SREBP1, were more resistant to NMDA toxicity than mock-treated control neurons.

The next step was unraveling the precise mechanism by which neuronal hyperactivation turns on SREBP1. SREBP1 ordinarily is inactive-trapped at the surface of the endoplasmic reticulum (ER) by a protein called insulin induced gene 1 (INSIG1). The UBC researchers found that NMDA caused ubiquitination and proteasomal degradation of INSIG1, which allowed SREBP1 to leave the ER and become active.

"In stroke, INSIG1 is degraded, liberating SREBP1," said Wang.

Wang thus reasoned that preventing INSIG1 degradation would also prevent SREBP1 activation. To test this hypothesis, the team developed a small peptide that interfered with INSIG1 ubiquitination and tested its effect on SREBP1 activation and neuron apoptosis.

In cultured rat neurons, treatment with the peptide prevented SREBP1 activation and neurotoxicity caused by NMDA compared with mock treatment. In live rats, animals given the peptides had lower levels of active SREBP1 and smaller ischemia-induced infarction than controls.

Altogether, the UBC study puts the SREBP1 regulation pathway in the center of the ischemic cascade that leads to neurotoxicity (see Figure 1, "New pathway to neuron death in stroke"). Results were published in Nature Medicine.
"This is a fascinating study that casts SREBP1 into an entirely new field-stroke and brain injury," said Andrew Brown, associate professor of biotechnology and biomolecular sciences at The University of New South Wales. "I think the use of the INSIG1 degradation-blocking peptide is the strongest data in the paper."

\section{INSIGnificant challenge}

Wang was upbeat about the prospects of delivering the INSIG1 stabilizer into the brains of stroke patients. "It's not difficult to make peptides that go into the brain," he said. "We use a transducing domain, a small sequence of 11 amino acids based on the HIV Tat protein. This fusion makes the peptide membrane permeable and able to pass through the blood brain barrier." ${ }^{4}$

Other researchers were more guarded about the peptide's prospects.

"How likely is it that a peptide with a transduction domain could be turned into a drug? That sounds very challenging," said Raymond Deshaies, professor of biology at the California Institute of Technology.

Deshaies, whose research focuses on proteasomal substrate recognition, noted that the precise target of Wang's peptide is an INSIG1specific ubiquitin ligase called gp78 that flags INSIG1 for degradation. Efforts to target proteins like gp78 have been largely unsuccessful because of the highly specific interaction between each ubiquitin ligase and its substrate. The details of this particular ligase-substrate pair are poorly understood, Deshaies added.

Russell DeBose-Boyd, associate professor of medical genetics at the University of Texas Southwestern Medical Center at Dallas, said 


\section{TARGETS \& MECHANISMS}

the UBC team's results were compelling but the INSIG1-stabilizing peptide's mechanism of action was difficult to interpret.

"It's the first time that I'm aware of where modulating INSIG1 levels has even been thought of for therapeutic purposes," said DeBose-Boyd. "From the SREBP1 point of view, the data are pretty convincing-when they knock down SREBP1, they can block cell death caused by NMDA. The point of contention is how the peptide works."

DeBose-Boyd, who has studied how INSIG1 binds to other components of cholesterol's metabolic machinery, suspects that Wang's peptide may do more than just block INSIG1 degradation, potentially blocking the proteasome's ability to degrade other substrates as well. Thus, he said Wang's peptide might have off-target effects.

Brown said any agent that blocks SREBP1 activation would have to be short-lived and used only in an acute setting. He noted that INSIG1 is required for proper cholesterol metabolism in the brain, which makes its own cholesterol rather than picking it up from the blood as other tissues do. Thus, "long-term inhibition of INSIG1 degradation could be disastrous, since the brain needs cholesterol" for normal neuron activity, he said.

DeBose-Boyd suggested that an alternative strategy for blocking SREBP1 activation could be fatostatin, a small molecule that works downstream of UBC's INSIG1 stabilizer and is being developed by a team at Baylor College of Medicine to treat lipid disorders (see Box 1, "Fat chance").

\section{Different strokes}

Regardless of the specific approach to preventing SREBP1 activation, new targets are a welcome addition to a therapeutic area in which most approaches have failed. ${ }^{5}$

Wang said his next goal is to test whether SREBP1 plays a role in other animal models of ischemic and hemorrhagic stroke. He also noted that NMDAR-mediated neurotoxicity is a factor in neurodegenerative diseases like amyotrophic lateral sclerosis (ALS) and Huntington's disease (HD) and said that his team is testing whether blocking SREBP1 activation could be useful in animal models of those diseases.

Wang has filed patents on the INSIG1 degradation-blocking peptide and is seeking industry partners.

Osherovich, L. SciBX 2(48); doi:10.1038/scibx.2009.1750

Published online Dec.17, 2009

\section{REFERENCES}

1. Taghibiglou, C. et al. Nat. Med.; published online Nov. 22, 2009; doi:10.1038/nm.2064

Contact: Yu Tian Wang, University of British Columbia, Vancouver, British Columbia, Canada e-mail: ytwang@interchange.ubc.ca

2. Ikonen, E. Nat. Rev. Mol. Cell Biol. 9, 125-138 (2008)

3. Adibhatla, R.M. et al. AAPS J. 8, E314-E321 (2006)

4. Borsello, T. et al. Nat. Med. 9, 1180-1186 (2003)

5. Flanagan, M. BioCentury 16(13), A1-A4; March 28, 2008

6. Kamisuki, S. et al. Chem. Biol.; published online Aug. 27, 2009; doi:10.1016/j.chembiol.2009.07.007

Contact: Motonari Uesugi, Kyoto University, Kyoto, Japan e-mail: uesugi@scl.kyoto-u.ac.jp Contact: Salih J. Wakil, Baylor College of Medicine, Houston, Texas e-mail: swakil@bcm.tmc.edu

7. Choi, Y. et al. J. Biol. Chem. 278, 7320-7324 (2003)

\section{COMPANIES AND INSTITUTIONS MENTIONED}

Baylor College of Medicine, Houston, Texas

California Institute of Technology, Pasadena, Calif.

The University of British Columbia, Vancouver, British Columbia, Canada

The University of New South Wales, Sydney, New South Wales, Australia

University of Texas Southwestern Medical Center at Dallas, Dallas, Texas 